\title{
Critical Chain Exercises
}

John Kevin Doyle, Benedictine University, USA

\begin{abstract}
Critical Chains project management focuses on holding buffers at the project level vs. task level, and managing buffers as a project resource. A number of studies have shown that Critical Chain project management can significantly improve organizational schedule fidelity (i.e., improve the proportion of projects delivered on time) and reduce average project duration, by reducing the unproductive time spent waiting for predecessor tasks to complete. Classroom exposition of Critical Chains requires mental models. Manufacturing examples, and particularly work in progress building up in front of specific bottleneck machines on the factory floor, have been effective examples for many years. Since many fewer practitioners have manufacturing experience than previously, the factory floor mental model does not work well with them. We describe a series of three "games" which have been used to teach managers and project managers about Critical Chains, and also used by the author in graduate Project Management courses the last two years. This paper describes more careful preparation for these simulations, to make them more useful in the classroom. Specifically, we more carefully define the setup, sequencing, instructions, and monitoring of each of the exercises, and develop a set of discussion questions for each exercise designed to lead the students to specific conclusions about the impacts of challenging objectives, multi-tasking, and the impact of randomness and batch size. We develop a pre-/post-test to assess student learning, and will use these to compare student learning before and after the modified improvements in the games.
\end{abstract}

Keywords: Critical Chains, simulation exercises, multi-tasking, random effects, classroom instruction

\section{INTRODUCTION}

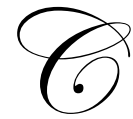

ritical Chains project management focuses on holding buffers at the project level vs. task level, and managing buffers as a project resource. A number of studies have shown that Critical Chain project management can significantly improve organizational schedule fidelity (i.e., improve the proportion of projects delivered on time) and reduce average project duration, by reducing the unproductive time spent waiting for predecessor tasks to complete. Eliyahu M. Goldratt's books (Goldratt 1994, 1997, 2004) were the seminal expositions of this theory, and remain important and persuasive. However, classroom exposition of Critical Chains requires more immediate mental models. Manufacturing examples, and particularly work in progress building up in front of specific bottleneck machines on the factory floor, have been effective examples for many years. Since many fewer practitioners have manufacturing experience than previously, the factory floor mental model does not work well with them.

Cooper (1999) and Roggenkamp (2005) describe a "game," developed at Lucent Technologies, which has been used to teach managers and project managers about Critical Chains. Kerzner (1984) and McCreery (2003) discuss ways of enabling student learning in project management classes, and of assessing that learning. Schuyler (2001) and Dinsmore (2006) discuss Critical Chains from the "handbook" perspective, and thus attempt to assist reader learning.

M. David Long used a series of Critical Chain simulation exercises (one of which was the Rizzo bead game) in training software development managers and project managers at Lucent Technologies and software, hardware, and marketing managers at Taqua. The author has used these simulation exercises with some success in graduate Project Management courses the last two years. The current work involves more careful preparation for these simulations, to make them more useful in the classroom. Specifically, we more carefully define the setup, 
sequencing, instructions, and monitoring of each of the exercises, and develop a set of discussion questions for each exercise designed to lead the students to specific conclusions about the impacts of challenging objectives, multitasking, and the impact of randomness and batch size. We develop pre- and post-tests to assess student learning, and use these to compare student learning before and after the modified improvements in the games.

\section{THE EXERCISES AND IMPROVEMENT OF EXERCISE EDUCATIONAL USEFULNESS}

The three exercises are:

1. Move three tennis balls around a team of students.

2. Move flat glass beads through a number of stages, with each student performing different mechanical tasks on the beads.

3. Move flat glass beads through stages simulating a sales / development / testing / production organization, introducing random stage productivity (using dice).

The first exercise is primarily designed to get the students up and moving, interacting as a team, and energized. The second and third exercises are directly modeling complex, multi-person projects.

Our long-term plan to improve the educational usefulness of these exercises is the following:

1. Develop explicit learning objectives for each exercise.

2. Develop a pre-test (also administered as post-test) to allow assessment of student learning, and therefore assessment of student learning improvement.

3. Assess learning using current exercise setup and discussion guidelines, administering pre-test and post-test.

4. Improve exercise setup and discussion guidelines.

5. Assess learning using revised exercise setup and discussion guidelines, administering pre-test and post-test.

We report in this paper on steps 1, 2 and 3 in the above program. Steps 4 and 5 will follow.

\section{EXERCISE 1}

Setup: Each team gets three tennis balls, marked 1, 2, and 3. The instructor instructs the team to stand in a circle. Each member of the team must touch each of the three balls in sequence $(1,2,3)$. The success metric is the time from when the first person touches ball 1, to when the last person touches ball 3 , and shorter time is better.

Exercise 1A: Each team is given a goal of 30 seconds. Since the teams (typically 4 to 7 students) have been arranged in a circle, the teams naturally pass the balls around the circle in sequence. Almost all teams easily meet the 30 second goal. No real thought or discussion is needed.

Exercise 1B: Same as Exercise 1A, but with a goal of 5 seconds. Since the teams were placed in circles in Exercise $1 \mathrm{~A}$, they tend to remain in a circle for Exercise 1B, and attempt to simply pass the balls faster. Teams can still achieve the goal, but it is much harder - any drops or fumbles will cause the team to miss the goal. Teams rarely change configuration in Exercise 1B - they simply try to go faster.

Exercise 1C: Same as Exercise 1B, but with a goal of 1 second. The teams now realize they must do something different. Most teams quickly come to have one team member hold the balls, and everyone else run their finger across the set of three balls.

Learning Objectives: There are three learning objectives for this exercise, and two observations which the students will have learned in other courses. The learning objectives are (1) an order of magnitude or better improvement in team performance on a complex task performed by an experienced team is possible, (2) doing what we are doing more carefully and precisely may not find order of magnitude improvements, and (3) how the assignment is given (i.e., the words, instructions, goals, monitoring, and reward systems) can greatly affect the ability of a team to find creatively different solutions. 
Related observations: Heterogeneous teams are more creative, and team heterogeneity is enhanced by heterogeneity in team members' gender, ethnic heritage, length of service, etc.

Pre- and Post-test questions:

1. In general, in a complex task performed by an experienced team, what improvement in team performance is possible? $10 \%, 50 \%, 2 \times, 10 \times$ (clearly there is no precise answer here, but what does your gut tell you)?

2. Is continuous, incremental team performance improvement the most effective way to improve team performance?

3. How critical are assignment phrasing, instructions, goals, monitoring, and reward systems to team performance? minimally, somewhat, significantly

\section{EXERCISE 2}

Setup: Each team gets 60 beads in a bowl, one plastic spoon, and three different colored plates. The beads are flower arranging glass beads - clear, about $1 / 2$ " round, and flat on one side and rounded on the other. Station 1 moves 20 beads from the bowl to first colored plate. Beads are only moved using the spoon, one at a time. Once the first colored plate has been completely processed, move it to Station 2. Repeat for the second and third colored plates.

Most of the specific directions are designed to make the exercise be complicated and take time. Many of the students spend a lot of time looking for ways around the instructions, rather than just playing the game.

Station 2 turns beads (which arrive randomly placed on the plate) round-side up, again using the spoon, one at a time. Some beads arrive at Station 2 already round-side up, so on average 30 beads need to be manipulated. Once all beads are round-side up, all beads are turned to flat-side up, using the spoon (one at a time). Once a plate has all beads flat-side up, move it to Station 3, and repeat for second and third colored plates.

Station 3 moves beads from the plates to the bowl. Beads are only moved using the spoon, one bead at a time. Once an entire plate is completely processed, the time is recorded according to the color of the plate. The process is then repeated for the second and third colored plates.

Exercise 2A: Each team is instructed to perform only five operations per colored plate, then move to another colored plate if there are any others in the team's queue. This allows the team to make progress on all tasks simultaneously (and to report progress to our managers).

Exercise 2B: Each team is instructed to complete all operations on each colored plate before moving to perform operations on the next plate.

So Exercise 2A forces multi-tasking, Exercise 2B forbids it. With rare exceptions, the teams perform better on Exercise 2B than $2 \mathrm{~A}$.

Learning Objectives: There are two learning objectives for this exercise, and three observations which the students will have learned in other courses. The learning objectives are (1) staff dedicated to one feature at a time - that is, not timesharing - produces better results, and (2) work-ahead is often counter-productive (e.g., resource may not be ready when needed, rework required), and therefore to be encouraged only when there are no other useful tasks for the resource.

Related observations: Idle time (if someone is not skilled to work at the current constraint) is used to perform rootcause analysis, construct tools, research industry developments, etc. This is typically measured in hours. Crosstraining is the key to getting larger resource/skill pools, which allows project progress even if we assign staff to one feature at a time. Cultural acceptance of this is key for management and staff. 


\section{Pre- and Post-test questions:}

4. Idle hands are the devil's workshop - if everyone is working on their tasks at all times, the organization as a whole is most productive.

5. Is work-ahead - working on your tasks in advance of deliveries of predecessor tasks - worthwhile or dangerous or both?

6. Have you heard the following, explicitly or implicitly?

- Individual staff members must realize that we expect you to walk and chew gum at the same time.

- The ability to multi-task is just another expectation of being a professional.

- The best multi-taskers are those that we will recognize and promote.

- Effective multitasking assures that we will always be making progress on all the items in our inbox.

\section{EXERCISE 3}

Introduction: We are developing new product releases via roadmaps, with pre-defined content and delivered according to our advanced gate planning processes. The roadmap defines seven features per release, with a release delivered every two months. Our customers will only accept a release every two months. It is important for us to deliver releases as quickly as possible (short cycle time) to meet market windows. The five different operations in our business involved with the definition, development, test and delivery of functionality are:

- $\quad$ Sales/Sales Engineering/Marketing

- Hardware/Software Development

- $\quad$ Feature Testing

- $\quad$ Systems Testing

- $\quad$ Customer Support/Manufacturing/Installation

Setup: Arrange the five members of each team in a row (at a long table). Each team member is given a single die. Begin with 70 beads in front of the first team member, representing the Sales function. The instructor will call out "Mark" every 30 seconds. Each team member throws a single die to determine how many beads can be moved from his/her input queue to his/her output queue. Record how many releases are made to the customer (i.e., full seven bead releases from the last station) at each "Mark".

Exercise 3A: The batch size is seven - if less than seven beads are in a team member's output queue at the "Mark," he/she needs to complete the batch, and then wait for the next "Mark" to move the batch to the next person's input queue. That is, you may not move less than a full batch to the next station. Also, the final station (Customer Support/Manufacturing/Installation) can only release full releases (seven beads) to the customer.

Exercise 3B: The batch size is one - whatever is in a person's output queue at "Mark" is moved to the next person's input queue. The final station can still only release full releases (seven beads) to the customer.

Learning Objectives: There are four learning objectives and four related observations. The learning objectives are (1) the small batch size exercise has a higher velocity - more finished releases per period, (2) the large batch size exercise has more "development inventory" - more beads build up everywhere, (3) the large batch size exercise likely requires more resource management (project, quality, priority), and (4) the small batch size exercise is more fun to work in - progress is more visible and satisfying.

Related Observations: "Development inventory" is dangerous, because Work in Progress (WIP) ages. The large batch size system is more susceptible to scope creep because WIP invites improvement. Clearly, higher velocity is business valuable. The large batch size system means more "development inventory," and that likely means lower quality, and therefore more in-field patching. 


\section{Pre- and Post-test questions:}

7. If we move work through our organization in batches (e.g., through sales, development, development testing, system testing, customer installation), which are more efficient (i.e., deliver more finished releases to customers per unit of time) - larger or smaller batches?

8. If we move work through our organization in batches, which have more work in progress (WIP) - larger or smaller batches?

9. If we move work through our organization in batches, which require more resource management - larger or smaller batches?

10. If we move work through our organization in batches, which is more fun to work in - larger or smaller batches?

\section{RESULTS}

In Fall 2009, one of our colleagues administered the pre- and post-test to his class, allowing assessment of student learning current exercise setup and discussion guidelines. The results were as follows ( $\mathrm{n}=18$ for the pre-test, $\mathrm{n}=19$ for the post-test).

1. In general, in a complex task performed by an experienced team, what improvement in team performance is possible? $10 \%, 50 \%, 2 \times, 10 \times$ (clearly there is no precise answer here, but what does your gut tell you)?

Results: in the pre-test, $51 \%$ answered $10 \%, 29 \%$ answered $50 \%, 18 \%$ answered $2 \times$, and $1 \%$ answered $10 \times$. In the post-test, the answers were $18 \%, 24 \%, 29 \%, 29 \%$-- a very significant shift (in fact, statistically significant at $\mathrm{p}=0.008$ ). Note that essentially every team attains $30 \times$ performance improvement on exercise 1 , so we would expect this shift (and maybe even a larger shift).

2. Is continuous, incremental team performance improvement the most effective way to improve team performance?

Results: in the pre-test, $58 \%$ said yes, $42 \%$ said no. In the post-test, $84 \%$ said yes, $16 \%$ said no. We find this counter-intuitive - the improvement in exercise 1 was not generated by continuous incremental performance, but rather a paradigm shift. We would have expected a smaller proportion of yeses in the post-test, not a larger. We observe that the shift is statistically significant $(\mathrm{p}=0.04)$.

3. How critical are assignment phrasing, instructions, goals, monitoring, and reward systems to team performance? minimally, somewhat, significantly

Results: In the pre-test, $2 \%$ said minimally, $8 \%$ somewhat, and $90 \%$ significantly. In the post-test, $4 \%$ said minimally, $21 \%$ somewhat, and $75 \%$ significantly. The shift is not statistically significant. Again, we find the shift somewhat counter-intuitive - the improvement in exercise 1 was only possible when the students carefully listened to the instructions, and realized they were not required to pass the balls around the circle.

4. Idle hands are the devil's workshop - if everyone is working on their tasks at all times, the organization as a whole is most productive.

Results: In the pre-test, $11 \%$ agreed and $89 \%$ disagreed. In the post-test, $42 \%$ agreed and $58 \%$ disagreed. This is a significantly significant change $(\mathrm{p}=0.01693)$. Since one of the lessons of exercise 2 was intended to be that everyone working on their tasks was not always productive, we clearly need to do better at getting this message to the students. This is probably best achieved with focused discussions after each exercise.

5. Is work-ahead - working on your tasks in advance of deliveries of predecessor tasks - worthwhile or dangerous or both? 
Results: In the pre-test, $11 \%$ felt that work-ahead was worthwhile, $6 \%$ dangerous, and $83 \%$ both worthwhile and dangerous. In the post-test, $26 \%$ felt that work-ahead was worthwhile, $11 \%$ dangerous, and $63 \%$ both worthwhile and dangerous. The shift is not statistically significant $(\mathrm{p}=0.08361)$. Since one of the lessons of exercise 2 was intended to be that work-ahead was not always productive, we clearly need to do better at getting this message to the students. This is probably best achieved with focused discussions after each exercise.

6. Have you heard the following, explicitly or implicitly?

a. Individual staff members must realize that we expect you to walk and chew gum at the same time.

b. The ability to multi-task is just another expectation of being a professional.

c. The best multi-taskers are those that we will recognize and promote.

d. Effective multitasking assures that we will always be making progress on all the items in our inbox.

Results: For 6a, pre-test was $26 \%$ explicitly, $50 \%$ implicitly, $24 \%$ no, post-test $34 \%$ explicitly, $34 \%$ implicitly, and $32 \%$ no. That is, there was a slight shift from $76 \%$ yes to $68 \%$ yes. For 6 b, pre-test yes was $88 \%$, post-test yes was $84 \%$. For $6 \mathrm{c}$, yes was $82 \%$ on the pre-test, $74 \%$ on the post-test. Finally, for $6 \mathrm{~d}$, $76 \%$ yes on the pre-test, $84 \%$ yes on the post-test. So, the pre- and post-test results were basically unchanged. This is as expected, because the questions ask about what the students have been told at work this should not be affected by the exercises.

7. If we move work through our organization in batches (e.g., through sales, development, development testing, system testing, customer installation), which are more efficient (i.e., deliver more finished releases to customers per unit of time) - larger or smaller batches?

Results: In the pre-test, $65 \%$ said smaller, in the post-test, $61 \%$. This is not a significant change (and is not statistically significant).

8. If we move work through our organization in batches, which have more work in progress (WIP) - larger or smaller batches?

Results: In the pre-test, $68 \%$ said larger, in the post-test, $71 \%$. This is not a significant change (and is not statistically significant). However, since one of the lessons of exercise 3 was intended to be that more WIP occurs with larger batch sizes, we clearly need to do better at getting this message to the students. This is probably best achieved with focused discussions after each exercise.

9. If we move work through our organization in batches, which require more resource management - larger or smaller batches?

Results: In the pre-test, $79 \%$ said larger, in the post-test, $71 \%$. This is not a significant change (and is not statistically significant). However, since one of the lessons of exercise 3 was intended to be that more resource management is needed with larger batch sizes, we clearly need to do better at getting this message to the students. This is probably best achieved with focused discussions after each exercise.

10. If we move work through our organization in batches, which is more fun to work in - larger or smaller batches?

Results: In the pre-test, $68 \%$ said larger, in the post-test, $61 \%$. This is not a significant change (and is not statistically significant).

\section{CONCLUSION}

The project described in this paper is just beginning. We have here described the Critical Chain simulation exercises, including the setup and two or three variant runs, the pre- and post-test questions we use to measure student learning, and related observations for the after exercise discussions. We have also described the first results 
from pre- and post-testing, and the first observations of where student learning is not as desired. We intend to continue administering the pre- and post-tests, to improve the exercise setup and discussion guidelines, and to assess learning using the revised exercise setup and discussion guidelines, administering pre-test and post-test to assess learning.

\section{AUTHOR INFORMATION}

Prof. John Kevin Doyle was born in New York, NY. He received his B.S. in Math from the University of Notre Dame and his M.A. and Ph.D. in Math and M.S. in Computer Science from Syracuse University. He was an Assistant Professor of Math and Computer Science at Emory University for five years, and worked at Bell Labs / AT\&T / NCR / Lucent for twenty years in a variety of general, line and staff management positions in software development, testing, project, program and product management and organizational development. He joined the Computer Science \& Information Systems department at Benedictine University in 2001, and the MBA department in 2004. His teaching interests include business, computer science, math, and organizational development. His research interests include the interaction of project management, business practice, and theory. He has published 18 papers in teaching, business, computer science, and math journals and proceedings.

\section{REFERENCES}

1. Cooper, Marjorie, Charlene Spoede Budd, and Lawrence Chonki (1999), Critical Chain Project Management, New Haven: Avraham Y. Goldratt Institute.

2. Dinsmore, Paul C. and Jeanette Cabanis-Brewin (eds.) (2006), The AMA Handbook of Project Management, $2^{\text {nd }}$ ed., New York: American Management Association.

3. Goldratt, Eliyahu M. and Jeff Cox (2004), The Goal: A Process of Ongoing Improvement, $3^{\text {rd }}$. ed., Great Barrington: North River Press.

4. Goldratt, Eliyahu M. (1994), It's Not Luck, Great Barrington: North River Press.

5. Goldratt, Eliyahu M. (1997), Critical Chain, A Business Novel, Great Barrington: North River Press.

6. Kerzner, Harold (1984), Methodologies for teaching project management in the classroom. Project Management Journal, 15 (August): 58-65.

7. McCreery, John K. (2003), Assessing the value of a project management simulation training exercise. International Journal of Project Management, 21(4): 233-242.

8. Roggenkamp, David B., Dave Park and Omer Tsimhoni (2005), A simulation model for facilitators of Tony Rizzo's bead game. Proceedings of the 37th Conference on Simulation; Session: Simulation education: teaching simulation beyond the traditional classroom, $2322-2328$.

9. Schuyler, John (2001), Risk and Decision Analysis in Projects, $2^{\text {nd }}$ ed., Newton Square: Project Management Institute. 
NOTES 